\title{
QUOTIENTS OF PROXIMITY SPACES
}

\author{
LOUIS FRIEDLER
}

\begin{abstract}
A characterization of the quotient proximity is given. It is used to find necessary and sufficient conditions for every proximity map on a space to be a topological quotient map. It is shown that a separated proximity space is compact iff every $p$-map on $X$ with separated range is a proximity quotient map.
\end{abstract}

Introduction. In 1959 Katetov [3] introduced proximity quotient maps. They have since been studied by Nachman [6], Poljakov ([7] and [8]), and Stone [9]. Although there are characterizations of proximity quotient maps in the literature [6], the only explicit formulation of the quotient proximity the author knows of is due to A. H. Stone, whose work appears in [10]. We give another characterization and use it to study mapping properties of proximity spaces.

Our notation will follow [10]. In particular, $A \subset \subset B$ will mean $A \not(X-B)$ and proximity maps will be called $p$-maps. $(X, \delta)$ will always denote a (not necessarily separated) proximity space. Given a completely regular space $X, \delta_{0}$ will represent the fine proximity: $A \phi_{0} B$ iff there is some $f \in C^{*}(X)$ such that $f(A)=0$ and $f(B)=1$.

\section{Characterization.}

1.1 Definition. If $\gamma$ and $\delta$ are two proximities on a set $X, \gamma$ is said to be finer than $\delta$ if $A \gamma B$ implies $A \delta B$. This will be written $\delta<\gamma$.

1.2 Definition. Let $f$ be a function from a proximity space $(X, \delta)$ onto a set $Y$. The quotient proximity is the finest proximity on $Y$ such that $f$ is a $p$-map. When $Y$ has the quotient proximity, $f$ will be called a $p$-quotient map.

1.3 TheOREM (STONE [9]). The quotient proximity is given by: $C \subset \subset D$ iff for each binary rational $s \in[0,1]$, there is some $C_{s} \subseteq Y$ such that $C_{0}=C$, $C_{1}=D$ and $s<t$ implies $f^{-1}\left(C_{s}\right) \subset \subset f^{-1}\left(C_{t}\right)$.

Presented to the Society, December 27, 1971; received by the editors March 8, 1972. AMS (MOS) subject classifications (1970). Primary 54E05, 54E10; Secondary $54 \mathrm{~B} 15$.

Key words and phrases. Quotient map, p-quotient map, p-open map.

(c) American Mathematical Society 1973 
1.4 Definition. Let $f$ be a function from a proximity space $(X, \delta)$ onto a set $Y$. Define $A \not^{*} B$ in $Y$ if there is a function $g: Y \rightarrow I$ such that $g(A)=0, g(B)=1$ and $g \circ f$ is a $p$-map.

\subsection{LEMMA. $\delta^{*}$ is a proximity.}

Proof. Clearly the axioms P1-P3 [10] hold and $A \delta^{*} B$ or $A \delta^{*} C$ easily imply $A \delta^{*}(B \cup C)$. Let $A \phi^{*} B$ and $A \not^{*} C$. We must show $A \phi^{*}(B \cup C)$. Let $g$ and $h$ map $Y$ to $I$ such that $g(A)=0, g(B)=1, h(A)=$ $0, h(C)=1$ and $g \circ f$ and $h \circ f$ are $p$-maps. Then $(g \cdot h)(A)=0$, $(g \cdot h)(B \cup C)=1$, and since the product of bounded real-valued $p$-maps is a $p$-map [3], $(g \cdot h) \circ f=(g \circ f) \cdot(h \circ f)$ is a $p$-map. Therefore, $A \not^{*}(B \cup C)$.

Now, let $A \not^{*} B$. Then there is a $g: Y \rightarrow I$ such that $g(A)=0, g(B)=1$ and $g \circ f$ is a $p$-map. The "strong axiom", P5, follows if $g$ is a $p$-map. Let $C \delta^{*} D$. If $\operatorname{cl}(g(C)) \cap \operatorname{cl}(g(D))=\varnothing$, there is a $p$-map $h: I \rightarrow I$ such that $h(\operatorname{cl}(g(C)))=0$ and $h(\operatorname{cl}(g(D)))=1$. But then $h \circ g \circ f$ is a $p$-map and $C \nexists^{*} D$-contradiction.

\subsection{THEOREM. $\delta^{*}$ is the quotient proximity.}

Proof. First, $f:(X, \delta) \rightarrow\left(Y, \delta^{*}\right)$ is a $p$-map. For if $A \delta^{*} B$, there is some $h: Y \rightarrow I$ such that $h(A)=0, h(B)=1$ and $h \circ f$ is a $p$-map. It follows that $f^{-1} h^{-1}(0) \not f^{-1} h^{-1}(1)$ and therefore $f^{-1}(A) \not f^{-1}(B)$.

Now, if $\delta^{\prime}$ is another proximity on $Y$ such that $f:(X, \delta) \rightarrow\left(Y, \delta^{\prime}\right)$ is a $p$-map, then $A \not^{\prime} B$ implies there is a $p$-map $h:\left(Y, \delta^{\prime}\right) \rightarrow I$ such that $h(A)=0$ and $h(B)=1$. Clearly, $A \phi^{*} B$ and hence $\delta^{*}$ is the finest proximity on $Y$ for which $f$ is a $p$-map.

1.7 Corollary. A function $g$ satisfying Definition 1.4 is continuous relative to the quotient topology on $Y$.

In the sequel $\delta^{*}$ will always denote the quotient proximity and $\zeta\left(\delta^{*}\right)$ the topology generated by $\delta^{*}$.

2. In this section we consider the question of when a proximity quotient map is a (topological) quotient map.

2.1 EXAMPLE. Let $X$ be the nonnegative real line with the usual proximity, identify $n$ and $1 / n$ for each positive integer $n$, and let $Y$ be the resulting set. Consider $U=\left[0, \frac{1}{2}\right) \cup \bigcup_{n>2}\{(n-1 / n, n+1 / n)\}$. Since $U=$ $f^{-1} f(U)$, where $f$ is the natural map, $f(U)$ is open in the quotient topology on $Y$. However, if $f(0) \subset \subset f(U)$, there must be some $V$ such that $f(0) \subset \subset$ $V \subset \subset f(U)$. Now, $\langle f(n)\rangle$ converges to $f(0)$, so there is an $N>2$ with $f(n) \in V$ for all $n \geqq N$. Thus, $\{f(n)\}_{n>. .} \delta^{*} Y-f(U)$. But clearly, $\{n\}_{n>N} \delta(X-U)$, 
so that $\{f(n)\}_{n>N} \delta^{*} Y-f(U)$. Therefore, $f(U)$ is not a $\zeta\left(\delta^{*}\right)$-neighborhood of $f(0)$ and $\zeta\left(\delta^{*}\right)$ is not the quotient topology even though $f^{-1}(y)$ is finite for all $y \in Y$.

2.2 THEOREM. Let $f:(X, \delta) \rightarrow\left(Y, \delta^{*}\right)$ be a 1-1 p-quotient map. Then (1) $A \delta^{*} B$ iff $f^{-1}(A) \delta f^{-1}(B)$, (2) $f$ is a p-isomorphism and (3) the topology induced by $\delta^{*}$ is the quotient topology.

Proof. (1) Let $f^{-1}(A) \not f^{-1}(B)$ and let $g: X \rightarrow I$ be a $p$-map such that $g\left(f^{-1}(A)\right)=0$ and $g\left(f^{-1}(B)\right)=1$. If $h: Y \rightarrow I$ is defined by $h(y)=g\left(f^{-1}(y)\right)$, then $h \circ f=g$ and so is a $p$-map. Hence, $A \not^{*} B$. (2) and (3) follow easily from (1).

2.3 THEOREM. If $\delta_{0}$ is the fine proximity on $X$ and $f:\left(X, \delta_{0}\right) \rightarrow\left(Y, \delta^{*}\right)$ is a p-quotient map, then $\zeta\left(\delta^{*}\right)$ is the quotient topology iff the quotient topology is completely regular.

Proof. Let the quotient topology, $\alpha$, be completely regular and let $\delta^{\prime}$ be any proximity compatible with $\alpha$. Since $\delta_{0}$ is the fine proximity, $f:\left(X, \delta_{0}\right) \rightarrow\left(Y, \delta^{\prime}\right)$ is a $p$-map so that $\delta^{\prime}<\delta^{*}$. Hence, $\alpha=\zeta\left(\delta^{\prime}\right) \subseteq \zeta\left(\delta^{*}\right)$. The opposite inclusion follows because every $p$-map is continuous.

Necessity is obvious.

2.4 THEOREM. Let $(X, \delta)$ be a proximity space. Then every proximity quotient of $X$ generates the quotient topology iff (1) $\delta$ is the fine proximity and (2) every (topological) quotient is completely regular.

Proof. Sufficiency follows from Theorem 2.3. For the necessity, assume every proximity quotient generates the quotient topology. If $\delta \neq \delta_{0}$, there are two sets $A$ and $B$ such that $A \delta B$ but $A \phi_{0} B$. That is, $A$ and $B$ are functionally separated. It follows that there is an open set $U$ such that $\bar{A} \subseteq U$ and $U \cap B=\varnothing$. Let $Y$ be the set formed by identifying $\bar{A}$ to a point, and give $Y$ the quotient proximity. Then, if $f$ is the natural map, $f(U)$ is an open neighborhood of $f(\bar{A})$ in the quotient topology. But $\bar{A} \delta B$ implies $f(\bar{A}) \delta^{*} f(B)$, so $f(\bar{A}) \in \operatorname{cl}_{\zeta_{\left(\delta^{*}\right)}} f(B)$. Clearly, $f(U)$ cannot be a $\zeta\left(\delta^{*}\right)$-neighborhood of $f(\bar{A})$-contradiction. Condition (2) above easily holds.

REMARK. Exactly the same proof will show that every separated proximity quotient generates the quotient topology iff ( $\left(1^{\prime}\right) \delta=\delta_{0}$ and $\left(2^{\prime}\right)$ every $T_{2}$ quotient is completely regular. MacDonald and Willard [5] have recently considered the problem of characterizing the spaces $X$ such that every $T_{2}$ quotient of $X$ is regular. 


\section{Mapping properties.}

3.1 THEOREM. Let $(X, \delta)$ be a separated proximity space. Then every $p$-map on $X$ with separated range is a p-quotient map iff $(X, \delta)$ is compact.

Proof. Assume $(X, \delta)$ is not compact. Then there is a cluster $\pi$ without a point. (See [4].) Let $p$ be any point in $X$ and define $\pi^{\prime}=\pi \cup \pi_{p}$, where $\pi_{p}$ is the cluster of all $A$ such that $A \delta p$. Finally, define $A \delta^{\prime} B$ iff either $A \delta B$ or both $A$ and $B \in \pi^{\prime}$.

We claim that $\delta^{\prime}$ is a proximity on $X$. Axioms (P1)-(P3) of [10] are easily verified. To prove (P4), observe that $\pi^{\prime}$, as the union of two clusters, inherits the following property of clusters:

(i) $B \cup C \in \pi^{\prime}$ iff $B \in \pi^{\prime}$ or $C \in \pi^{\prime}$.

Now, let $A \delta^{\prime}(B \cup C)$. If $A \delta(B \cup C)$, we are done, so assume $A \not B(B \cup C)$. Then we must have $A \in \pi^{\prime}$ and $(B \cup C) \in \pi^{\prime}$. It follows from (i) that $A \delta^{\prime} B$ or $A \delta^{\prime} C$. The reverse implication in (P4) also follows from (i).

To prove (P5), let $A \not^{\prime} B$. Then either $A \notin \pi^{\prime}$ or $B \notin \pi^{\prime}$. By symmetry we need only consider the case where $A \notin \pi^{\prime}$. Then, $A \not \varnothing B, A \not p$, and since $A \notin \pi, A \not P$ for some $P \in \pi$. Thus, there exist disjoint sets $U$ and $V$ such that $A \not(X-U)$ and $(B \cup P \cup\{p\}) \not(X-V)$. But $(X-V) \notin \pi$ since $(X-V) \not P$ and $P \in \pi$, and $(X-V) \notin \pi_{p}$ since $(X-V) \not p$, so $(X-V) \notin \pi^{\prime}$. Since $A$ and $(X-V)$ are not in $\pi^{\prime}, A \phi^{\prime}(X-U)$ and $(B \cup P \cup\{p\}) \phi^{\prime}(X-V)$, and (P5) is satisfied.

Note that $\delta^{\prime}$ is easily separated and $\delta^{\prime}<\delta$. It follows from our assumption that $i:(X, \delta) \rightarrow\left(X, \delta^{\prime}\right)$ is a one-to-one $p$-quotient map, and so, by Theorem 2.2, $\delta=\delta^{\prime}$. This contradicts the definition of $\delta^{\prime} ;$ therefore, $(X, \delta)$ is compact.

Conversely, assume $(X, \delta)$ is compact and let $f$ be a $p$-map from $X$ onto a separated proximity space $\left(Y, \delta^{\prime}\right)$. Then the quotient topology is $T_{31}$ and $\delta$ is the fine proximity on $X$. It follows from Theorem 2.3 that $\zeta\left(\delta^{*}\right)$ is the quotient topology $\alpha$. Since $f:(X, \zeta(\delta)) \rightarrow\left(Y, \zeta\left(\delta^{\prime}\right)\right)$ is a continuous function on a compact set, $\zeta\left(\delta^{\prime}\right)=\alpha$. But since $(Y, \alpha)$ is compact and $T_{2}$, it has a unique compatible proximity [10]; hence, $\delta^{\prime}$ is equivalent to $\delta^{*}$, the quotient proximity.

REMARK. It is well known that a $T_{3 \hat{2}}$ space $X$ is locally compact iff it has a minimal compatible proximity. If we consider a separated proximity on a set $X$ to be minimal separated if $\delta^{\prime}<\delta$ and $\delta^{\prime}$ separated imply $\delta^{\prime}=\delta$, then we have the following

3.2 CoROllaRY. Let $(X, \delta)$ be a separated proximity space. Then $(X, \delta)$ is compact iff $\delta$ is minimal separated on $X$.

3.3 THEOREM. Let $Y$ be any completely regular space and $\delta^{\prime}$ any compatible proximity. Then $\delta^{\prime}$ is the fine proximity on $Y$ iff for all proximity 
spaces $(X, \delta)$ and all p-maps $f:(X, \delta) \rightarrow\left(Y, \delta^{\prime}\right)$ onto $Y$ such that $\zeta\left(\delta^{\prime}\right)$ is the quotient topology induced by $f$, then $f$ is a p-quotient map.

Proof. Let $\delta^{\prime}=\delta_{0}$, the fine proximity on $Y$, and let $f:(X, \delta) \rightarrow\left(Y, \delta_{0}\right)$ be a $p$-map onto $Y$ such that $\zeta\left(\delta_{0}\right)$ is the quotient topology $\alpha$. Then $\delta_{0}<\delta$ * where $\delta^{*}$ is the quotient proximity. If $A \phi^{*} B$ there is some $g: Y \rightarrow I$ such that $g$ is continuous relative to $\alpha=\zeta\left(\delta^{\prime}\right)$ and $g(A)=0, g(B)=1$ and $g \circ f$ is a $p$-map. Therefore $A \phi_{0} B$ so that $\delta_{0}=\delta^{*}$ and $f$ is $p$-quotient.

For the converse, consider $i:\left(Y, \delta_{0}\right) \rightarrow\left(Y, \delta^{\prime}\right)$ and apply Theorem 2.2.

If $f:(X, \delta) \rightarrow\left(Y, \delta^{*}\right)$ is a $p$-quotient map, we shall write $\delta^{*}=\delta / f$.

3.4 CoRollary. Let $(X, \delta)$ be a proximity space and $(Y, \delta / f)$ a pquotient such that the quotient topology is completely regular. Then $\delta / f$ is the fine proximity on the quotient topology iff $\delta / f=\delta_{0} / f$, where $\delta_{0}$ is the fine proximity on $X$.

Proof. Sufficiency is easy while necessity follows from the above theorem.

Poljakov ([7] and [8]) defined a $p$-map to be $p$-open if $A \subset \subset B \Rightarrow$ $f(A) \subset \subset f(B)$. The proof of the next theorem is routine and thus omitted.

3.5 THEOREM. Let $f$ map a proximity space $(X, \delta)$ onto a proximity space $\left(Y, \delta^{\prime}\right)$. Then $f$ is p-open iff $f(A) \delta^{\prime} B$ implies $A \delta f^{-1}(B)$.

3.6 THEOREM. Let $(X, \delta)$ be a proximity space such that every p-quotient map on $X$ is p-open. Then every p-quotient generates the quotient topology. Consequently, $\delta$ is the fine proximity on $X$.

Proof. If $X$ has a $p$-quotient $(Y, \delta / f)$ which does not generate the quotient topology, then $f$ is not open, hence not $p$-open-contradiction.

There do not seem to be reasonable sufficient conditions on $X$ such that every $p$-quotient on $X$ is $p$-open. For example, if $X=[0,2]$ and $\left[\frac{1}{2}, \frac{3}{2}\right]$ is identified to a point, the natural map is $p$-quotient but not open. A related topological problem is to characterize the topological spaces $X$ such that every topological quotient map on $X$ is open. See MacDonald and Willard [5] for similar problems.

Proximity bi-quotient maps will be considered in a forthcoming paper.

ACKNOWLEDGEMENTS. (1) This paper contains part of the author's $\mathrm{Ph}$.D. Thesis written at the University of Alberta under the supervision of Dr. Stephen Willard.

(2) The author would like to thank the referee for a suggestion which implified the proof of Theorem 3.1. 


\section{BIBLIOGRAPHY}

1. C. H. Dowker, Mappings of proximity structures, General Topology and its Relations to Modern Analysis and Algebra (Proc. Sympos., Prague, 1961), Academic Press, New York; Publ. House Czech. Acad. Sci., Prague, 1962, pp. 139-141. MR 26 \#4312.

2. J. R. Isbell, Uniform spaces, Math. Surveys, no. 12, Amer. Math. Soc., Providence, R.I., 1964. MR 30 \#561.

3. M. Katětov, Über die Berührungsräume, Wiss. Z. Humboldt-Univ. Berlin Math.Natur. Reihe 9 (1959/60), 685-691. MR 32 \#1672.

4. S. Leader, On clusters in proximity spaces, Fund. Math. 47 (1959), 205-213. MR 22 \#2978.

5. S. MacDonald and S. Willard, Domains of paracompactness and regularity, Canad. J. Math. (to appear).

6. L. J. Nachman, Quotients of complete proximity spaces, Bull. Acad. Polon. Sci. Ser. Sci. Math. Astronom. Phys. 18 (1970), 17-20. MR 42 \#1058.

7. V. Z. Poljakov, Open mappings of proximity spaces, Dokl. Akad. Nauk SSSR 155 (1964), 1014-1017=Soviet Math. Dokl. 5 (1964), 545-548. MR 30 \#2460.

8. - On proximity open mappings of metric spaces, Dokl. Akad. Nauk SSSR 186 (1969), 1012-1015 =Soviet Math. Dokl. 10 (1969), 705-708. MR 40 \#6493.

9. A. H. Stone, Lecture notes, University of Rochester, Rochester, N.Y., 1967 (unpublished).

10. S. Willard, General topology, Addison-Wesley, Reading, Mass., 1970. MR 41 \#9173.

Department of Mathematics, University of Alberta, Edmonton, Alberta, CANADA

Current address: Department of Mathematics, University of Texas, Austin, Texas 78712 